\title{
NOTA SOBRE LOS AUTORES
}

\section{ABOUT THE AUTHORS}

\section{CAmilo Fernández Cozman}

Camilo Fernández Cozman (Lima, 1965) es Miembro de Número de la Academia Peruana de la Lengua y profesor de la Universidad de San Marcos, de la Universidad San Ignacio de Loyola y de la Universidad de Lima. Ha sido conferencista en Madrid, Salamanca, Burdeos, Roma, Florencia, Siena, Bérgamo, Bolonia, Rímini y Zurich. Ha publicado Las insulas extrañas de Emilio Adolfo Westphalen (ensayo,1990), Ritual del silencio (1995), Las huellas del aura. La poética de J.E. Eielson (1996), Raúl Porras Barrenechea y la literatura peruana (2000), Rodolfo Hinostroza y la poesía de los años sesenta (2001), El cántaro y la ola. Una aproximación a la poética de Octavio Paz (2004), La soledad de la página en blanco (2005), La poesía hispanoamericana y sus metáforas (Murcia, 2008), Mito, cuerpo y modernidad en la poesía de José Watanabe (2009), La poesía es como el aroma (Buenos Aires, 2009), Casa. Cuerpo. La poesía de Blanca Varela frente al espejo (2010), Sujeto, metáfora, argumentación (2011) y César Moro, ¿un antropófago de la cultura? (2012).

\section{Patricia García}

Patricia García es doctora en literatura comparada por la Dublin City University (Irlanda) con la tesis titulada The Architectural Void: Space as Transgression in Postmodern Short Fiction of the Fantastic (1974-2010). Es profesora en Trinity College Dublin y en la Dublin City University. Es, además, profesora asociada en el Instituto Cervantes de Dublín (donde imparte clases sobre el relato corto contemporáneo en España y Latinoamérica, y sobre escritura creativa), y también colabora como docente en cursos de formación de profesores de ELE. Asimismo, es miembro del Grupo de Estudios sobre lo Fantástico (GEF) de la Universidad Autónoma de Barcelona. Ha sido profesora invitada en la Lebanese American University (Beirut, Líbano), y ha presentado parte de su investigación en varias conferencias internacionales sobre el espacio y lo fan- 
tástico. Entre sus publicaciones se puede destacar su ensayo sobre las figuras del desplazamiento en Thomas Bernhard y Agota Kristov (2010).

\section{Sergio Hernández Roura}

Sergio Hernández Roura (México D.F., 1983) es escritor mexicano. Es licenciado en Letras Hispánicas por la UNAM y maestro en Literatura Comparada por la Universidad Autónoma de Barcelona, institución en la que realiza actualmente sus estudios de doctorado (La recepción de Edgar Allan Poe en México Siglo XIX). Formó parte de la redacción de la revista Artes de México, fue editor de Endora Ediciones y ha sido becario del FONCA (Fondo Nacional para la Cultura y las Artes, periodos 2010-2011, 2012-2013).

\section{Audrey Louyer Davo}

Audrey Louyer Davo está realizando su tesis doctoral sobre la noción de «pasaje/paso» en la literatura fantástica peruana desde 1950 en la Universidad de Reims Champagne-Ardennes. Realizó el Master II Recherche de la Universidad de Reims con la tesina titulada: «Réflexions et réfractions du fantastique dans La Piedra en el agua de Harry Belevan». Su línea de investigación es la literatura latinoamericana fantástica y actualmente trabaja como docente en la enseñanza secundaria.

\section{Marisa Martins Gama-KhaliL}

Possui Mestrado em Letras: Literaturas de Língua Portuguesa pela Universidade Estadual Paulista Júlio de Mesquita Filho/Assis (1994) e Doutorado em Estudos Literários pela Universidade Estadual Paulista Júlio de Mesquita Filho/Araraquara (2001). Trabalhou de 1987 a 2006 na Universidade Federal de Rondônia. Atualmente, é professora, nível Associado I, da Universidade Federal de Uberlândia, onde atua na graduação em Letras e no Mestrado em Teoria Literária. É também professora colaboradora do Mestrado em Letras: Linguagem e Identidade da UFAC - Universidade Federal do Acre. Seu projeto «Representações espaciais do horror na narrativa fantástica brasileira dos séculos XX e XXI» é contemplado com a bolsa de Produtividade em Pesquisa - CNPq. Tem livros, artigos e capítulos de livro publicados, com ênfase nas reflexões sobre o espaço ficcional e sobre a narrativa fantástica; bem como nas relações plausíveis entre Teoria Literária e Análise do Discurso. 


\section{Daniel-Henri Pageaux}

Daniel-Henri Pageaux (Paris, 1939). Il est élu en 1975 à la Sorbonne Nouvelle professeur dans une chaire de littérature comparée (littératures ibériques), il est aussi spécialiste des littératures francophones (Afrique, Amériques). De 1977 à 1979, puis de 1981 à 1983 il a été Président de la SFLGC/Société française de Littérature générale et comparée. Il a été Directeur de l'UFR de Littérature générale et comparée et Directeur du CRA/Centre de recherches africaines (Paris I, II, V), de 1987 à 1990. Il est membre correspondant de l'Académie des sciences/lettres de Lisbonne. Il est co-directeur de la Revue de Littérature Comparée.

\section{Nicola Pasqualicchio}

Nicola Pasqualicchio è ricercatore di Storia del teatro e dello spettacolo presso l'Università di Verona. I suoi interessi riguardano in particolare la drammaturgia e le teorie teatrali europee dei secoli XIX e XX: in tale ambito ha pubblicato un volume sul teatro di Beckett (Il sarto gnostico, 2006) e vari saggi su Artaud, sul quale ha anche in preparazione una monografia, oltre ad articoli su Delsarte, Ibsen, D’Annunzio, Savinio, Pirandello, Genet: si è inoltre occupato della recitazione fra teatro e cinema e di alcuni aspetti tematici del teatro shakespeariano. Negli ultimi anni, coniugando i propri precedenti interessi sulla letteratura fantastica (che avevano tra l'altro portato alla pubblicazione, nel 1993, del volume Maladea sul ritorno degli dei antichi come fantasmi nella narrativa ottocentesca) con quelli relativi alle arti sceniche, ha indirizzato le proprie ricerche sul teatro fantastico, in particolare dell'Ottocento e inizio Novecento, sia con riflessioni storiche ed estetiche di carattere generale (come quella apparsa nel 2012 sulla rivista Pygmalion, o l'introduzione al volume collettaneo da lui curato La meraviglia e la paura. Il fantastico nel teatro europeo, in corso di stampa), sia con analisi specifiche di alcuni testi drammatici (in particolare Le Vampire di Alexandre Dumas père e alcuni drammi italiani ugualmente incentrati sulla figura del vampiro, e Il cuore di cera, progetto di balletto fantastico del pittore simbolista Alberto Martini).

\section{DOLORES PHILLIPPs-LóPEZ}

Doctora en literatura hispanoamericana por la Université de Genève (Suiza), es actualmente docente e investigadora en la Université de Lausanne (Suiza) y encargada de cursos en la Université de Genève. Ha publicado diversos artícu- 
los sobre lo fantástico (siglo XIX y principios del XX) y en 2003 editó Cuentos fantásticos modernistas de Hispanoamérica (Cátedra, Madrid).

RubÉn SÁNCHEZ TRIGOS

Rubén Sánchez Trigos (Madrid, 1979) es profesor de cine en U-Tad (Madrid), y miembro del grupo de investigación «Imaginarios, Estudios Narrativos en Medios y Artes Audiovisuales». Actualmente trabaja en su tesis doctoral sobre el zombi en el cine español. Ha publicado la novela Los huéspedes (Drakul, 2009), Finalista del Premio Drakul de Novela, así como diversos cuentos en distintas antologías. Es co-guionista de El intruso, nominado al Goya al Mejor Cortometraje de Ficción 2005.

\section{Alejo Steimberg}

Alejo Steimberg es licenciado en letras por la Universidad de Buenos Aires. Ha publicado artículos académicos y periodísticos en publicaciones en Argentina, Bélgica, Francia, España y Países Bajos. Actualmente redacta su tesis sobre la presencia de lo fantástico en las novelas de ciencia ficción de Philip K. Dick (Universidad de Extremadura). Vive en Bélgica.

\section{SILVIA ZANGRANDI}

Silvia Zangrandi has been working as a Ricercatore (Assistant Professor) of Contemporary Italian Literature at Università IULM - Milano (Italy) since 2005. She received her Ph. D. in Comparative studies. Her publications include studies about Dino Buzzati, Italo Calvino, Primo Levi, Giorgio Mangane1li, Luigi Meneghello, Eugenio Montale, Anna Maria Ortese, Cesare Pavese; the volumes A servizio della realtà (2003) on the journalistic novel in Italy, Lingua e attualità letteraria (2006) about the evolution of the Italian language in the XX century, Pagine infestate (2007) about the evolution of the topos of the ghost and Cose dell'altro mondo. Percorsi nella letteratura fantastica italiana del Novecento (2011) about the italian fantastic literature of XX century. Her current researches are focusing on: comparative studies concerning the fantastic literature of the XX century; the relationship between literature and journalism; the Italian narrative of the second half of the XX century, with particular attention to the linguistic solutions adopted by the writers. 